\title{
Characterization of a Rhodium(III)-Imine-Orthometalated Imine Complex; Reversible C-H Activation of a Coordinated Imine
}

\author{
Maria B. Ezhova, Brian O. Patrick, and Brian R. James* \\ Department of Chemistry, University of British Columbia, 2036 Main Mall, Vancouver, \\ B.C. V6T 1Z1, Canada.
}

\section{Supporting Information}

Scheme S1. Formation of complexes with the $\mathrm{RN}=\mathrm{CR}$ ' $(\mathrm{Ph})$ imines, and derived orthometalated complexes (adapted from ref. 2) $\mathrm{P}=\mathrm{PPh}_{3} ; \mathrm{S}=$ solvent (acetone or $\mathrm{MeOH})$.

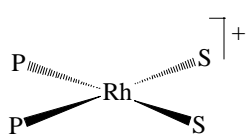

(1) or

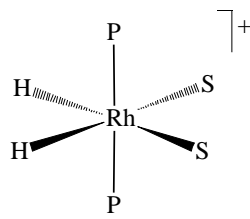

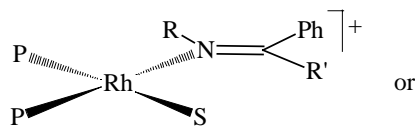

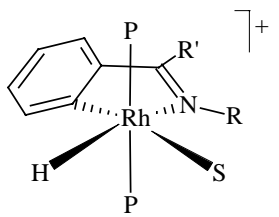




\section{Details on the refinement of the X-ray crystallographic data for complex 2 (see also the CIF file)}

The SQUEEZE function found in PLATON generates a new reflection file that is corrected to account for any electron density found in void spaces. One phenyl ring was found to be disordered in two orientations. The atoms of this phenyl ring were refined isotropically, while all other non-hydrogen atoms were refined anisotropically. H-atoms are included but not refined. Post-refinement difference maps were generated and searched for residual electron density consistent with either imine-hydrogens or metal hydrides. One difference peak consistent with a Rh-hydride was located and isotropic refinements on this $\mathrm{H}$-atom gave reasonable results. Difference peaks consistent with imine-hydrogens were located; however, subsequent isotropic refinements proved to be unstable and the positions of these "H-atoms" shifted away from their associated $\mathrm{N}$ atoms. Data correction by SQUEEZE, and more importantly the relatively weak scattering, likely play a role in the uncertainty of the imino-hydrogen positions; however, the bond lengths and angles associated with the ligands are consistent with the presence of imines. The final cycle of full-matrix least-squares refinement was based on 10723 reflections and 597 variable parameters. 